\title{
Research On A Multi-Missile Distributed Cooperative Guidance Method Based On Dynamic Network
}

\author{
Jiyang Dai, a , Wenhua Li ${ }^{1, b}$,Baojian Yang ${ }^{1, c}$,Junqiang Xiong ${ }^{1, d}$ \\ ${ }^{1}$ Unit 696, Honggutan District, Nanchang Prov., P.R.China.

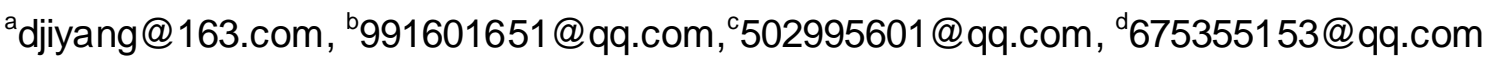

Keywords: Multi-Missile Cooperative Control Dynamic Networks, Terminal Guidance Attack, Distributed Cooperative Guidance Method

Abstract.To solve the problem of multi-missile cooperative control for dynamic networks, a method of dynamic network analysis based on graph theory is proposed, the multi-missile flight states are analyzed with the connectivity of missile network; the variable time is added to the traditional proportional navigation guidance method to improve the distributed cooperative guidance method. The results show that the missile network have good invulnerability when attacked; The angle and time of the missile can achieve the cooperative requirement in the terminal guidance attack. The effectiveness and feasibility of the missile network coordinate guidance control method is verified by the numerical simulation results.

\section{Introduction}

The control of multi-missile cooperative guidance is described as autonomous collaborative completion of one or more common guidance tasks with coordination of data through sharing information and mutual influence between missiles [1]. With the development of the war situation, the implementation of multiple attacks has become the main form of modern warfare. That contains two aspects of the content, one is the multi-missile formation flight, the other is a number of missiles attacking target at the same time [2]. A defense of enemy firepower in different directions of attack at the same time can make its interception ability significantly reduced, that will effectively improve the missile formation damage and penetration ability.

In this study, the communication topology of missile network is considered, and the goal of multi-missile distributed cooperative saturation attack is achieved. Formation cooperative guidance strategy, which is advantageous to network invulnerability and network connected to recover and keep, can overcome the adverse effects of the operational environment network dynamic characteristics.

\section{Distributed cooperative guidance method}

Multiple missiles emit from different directions, even in different times to achieve the effect of concentrated saturated attack on the target at the same time or with the little error of time by the fusion of information [3-5]. Because of the attack time which the missile estimating independently determined by the uniform coordination, the human intervention greatly reduced. With strong autonomy, this kind of way of cooperative attack can actualize the true sense of the cooperative guid ance. Fig. 1 shows that the battleground of six missiles salvo the same target. 


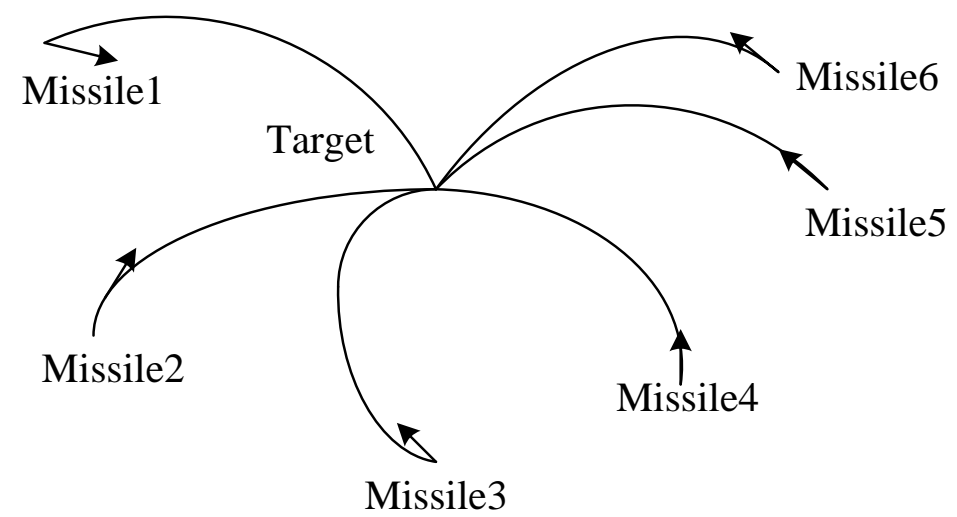

Fig. 1 the sketch of $\mathrm{N}$ missiles salvo the same target

The structure of cooperative guidance is shown in Fig.2:

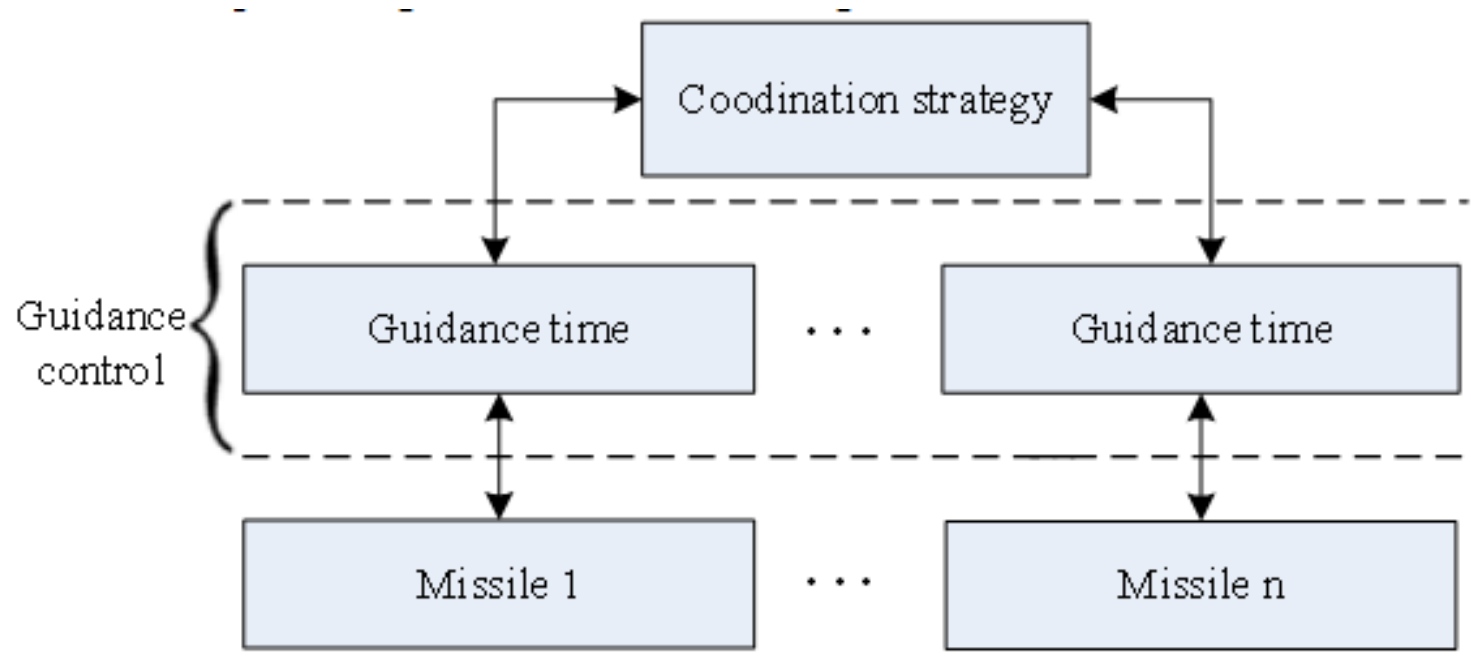

Fig. 2 The structure sketch of distributed cooperative guidance

The paper puts forward a way of attack time control in cooperative combat to improve the missile cooperative penetration and precision strike capability. The relative motion relationship between the $i^{\text {th }}$ missile and the target is shown in Fig.3.

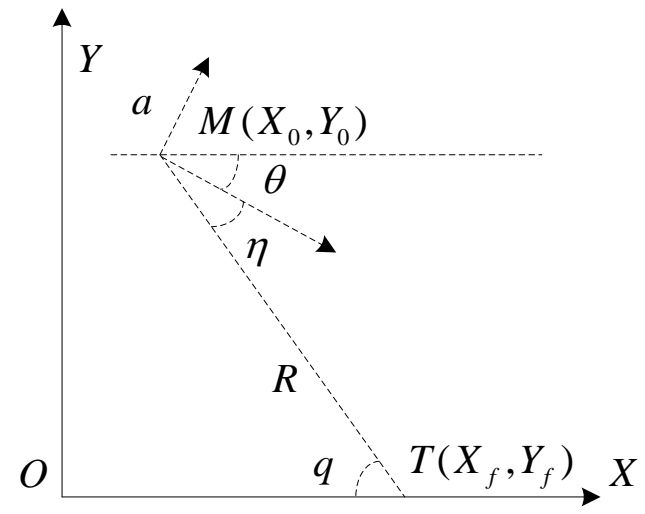

Fig.3 Relative motion relationship between the missile and the target

Where, $M$ and $T$ represent the missile and target, respectively; $R$ described as the distance of the missile and target; $X, Y$ and $\theta$ expressed as horizontal, vertical and angular velocity, respectively; The target can be regarded as a stationary at $\left(X_{f}, Y_{f}\right)$ with respect to the high-speed motion of the missile. Supposed that missile velocity is $V$, subscript 0 and $f$ represent the starting and terminal time respectively, $\eta$ is aspect angle of seeker, $q$ is high-low angle of sight between missile and target, $T_{f}$ is the expected guidance time, $a$ is normal acceleration of missile. 
It is assumed that the seeker tracking target is ideal, and no time delay, the missile's motion equation [6] is shown in the equation set (1).

$$
\left\{\begin{array}{l}
\dot{X}=V \cos \theta, X(0)=X_{0}, X\left(T_{f}\right)=X_{f} \\
\dot{Y}=V \sin \theta, Y(0)=Y_{0}, Y\left(T_{f}\right)=Y_{f} \\
q=\theta+\eta \\
\dot{\theta}=a / V, \theta(0)=\theta_{0} \\
\dot{R}=-V \cos \eta \\
R \dot{q}=V \sin \eta
\end{array}\right.
$$

Multi-missile system setting attack time needs to meet the constraint prerequisite showed in the inequality formula (2).

$$
T_{d} \geq \max \left\{\stackrel{*}{T}_{i}\right\} \quad i \in\{1,2, \ldots, n\}
$$

$n$ is the number of missile, the $\mathrm{i}^{\text {th }}$ missile's estimating attack time is $\stackrel{*}{T_{i}}$.

On the basis of pure proportional navigation guidance (PNG) [7], a time correction term is added, which can make the missile hit the target at a predetermined time. That expressed as following equation (3).

$$
a=a_{p}-\frac{60 V^{5}}{a_{p} R^{3}}\left(T_{d}-\stackrel{*}{T}\right)
$$

The first term $a_{p}=N V \dot{\theta}$ is the control quantity computed by PNG, $N$ is navigation ratio, $\theta$ is aspect angle; the second term is the correction term for controlling lead time, $T_{d}$ is the predetermined time, the method of estimation of the guidance time of proportional navigation guidance law is adopted in Ref.[8], the estimation of the guidance time ${ }^{*}$ is calculated by the following equation [9]:

$$
T^{*} \approx \frac{R}{V}\left(1+\frac{(\theta-q)^{2}}{10}\right)
$$

$\xi$ is used as a coordination variable to control the guidance time, for controllable time variable(expected guidance time $T_{d}$ ), coordination variable $\xi$ is regard as expected guidance time $T_{d}$, that is : $\xi=T_{d}$. Supposed that there is $n$ missiles to be involved in the cooperative guidance task, with the same expected guidance time, missiles can be able to hit the target cooperatively under the guidance of ITCG in that process [10].

The control quantity for the $i^{\text {th }}$ missile can be calculated as $u_{i}=a_{p i}-a_{i}\left(\xi-T_{d i}\right)$ from the formula (3), Where, $a_{i}=60 V_{i}^{5} /\left(a_{p i} R_{i}^{3}\right)$. Sub optimal value solution of coordination variable can be solved out by the following equation:

$$
\xi=\sum_{i=1}^{n} a_{i}^{2} T_{i} / \sum_{i=1}^{n} a_{i}^{2}
$$

Notwithstanding $\xi$ is not strictly optimal solution, it not only indicates the weighted average of the remaining estimate time of each missile, it can also be considered as expected time value that obtained through negotiation of multiple missiles.

\section{Simulation verification}

Simulation analysis of network connectivity. Before the missile attack cooperatively, first, we analyze the network connectivity of six missiles and determine missile network topology. After that, to compute the Laplace matrix of the network and compute relative error and absolute error by 
setting the initial state of the missile. Finally, using the numerical analysis algorithm of Runge Kutta to get the missile state and mapping. The topology structure of the $6^{\text {th }}$ missile being not connected with other 5 missile networks is shown in Fig.4.

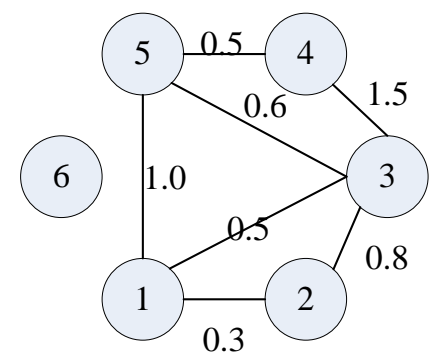

Fig.4 The topology structure of missile network after attacked

The structure of the system is

$$
\left[\begin{array}{l}
\dot{X}_{1}(t) \\
\dot{X}_{2}(t) \\
\dot{X}_{3}(t) \\
\dot{X}_{4}(t) \\
\dot{X}_{5}(t) \\
\dot{X}_{6}(t)
\end{array}\right]=-\left[\begin{array}{cccccc}
1.8 & -0.3 & -0.5 & 0 & -1.0 & 0 \\
-0.3 & 1.1 & -0.8 & 0 & 0 & 0 \\
-0.5 & -0.8 & 3.4 & -1.5 & -0.6 & 0 \\
0 & 0 & -1.5 & 2 & -0.5 & 0 \\
0 & 0 & 0.6 & -0.5 & 0.5 & 0 \\
0 & 0 & 0 & 0 & 0 & 0
\end{array}\right]\left[\begin{array}{c}
X_{1}(t) \\
X_{2}(t) \\
X_{3}(t) \\
X_{4}(t) \\
X_{5}(t) \\
X_{6}(t)
\end{array}\right]
$$

The topology structure of six missiles completely connected is shown in Fig.5:

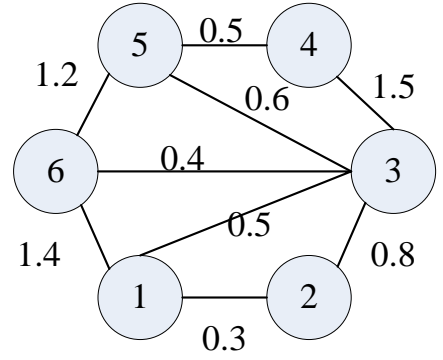

Fig.5 The topology structure of missile network completely connected The structure of the system is

The initial state of missile is set as

$$
\left[\begin{array}{c}
\dot{X}_{1}(t) \\
\dot{X}_{2}(t) \\
\dot{X}_{3}(t) \\
\dot{X}_{4}(t) \\
\dot{X}_{5}(t) \\
\dot{X}_{6}(t)
\end{array}\right]=-\left[\begin{array}{cccccc}
2.2 & -0.3 & -0.5 & 0 & 0 & -1.4 \\
-0.3 & 1.1 & -0.8 & 0 & 0 & 0 \\
-0.5 & -0.8 & 3.8 & -1.5 & -0.6 & -0.4 \\
0 & 0 & -1.5 & 2 & 0.5 & 0 \\
0 & 0 & 0.6 & -0.5 & 2.3 & -1.2 \\
-1.4 & 0 & -0.4 & 0 & -1.2 & 3
\end{array}\right]\left[\begin{array}{c}
X_{1}(t) \\
X_{2}(t) \\
X_{3}(t) \\
X_{4}(t) \\
X_{5}(t) \\
X_{6}(t)
\end{array}\right]
$$

$$
\left[\begin{array}{lllllll}
X_{1}(0) & X_{2}(0) & X_{3}(0) & X_{4}(0) & X_{5}(0) & X_{6}(0)
\end{array}\right]^{T}=\left[\begin{array}{llllll}
-15 & 98 & 38 & 12 & 65 & -5
\end{array}\right]^{T}
$$



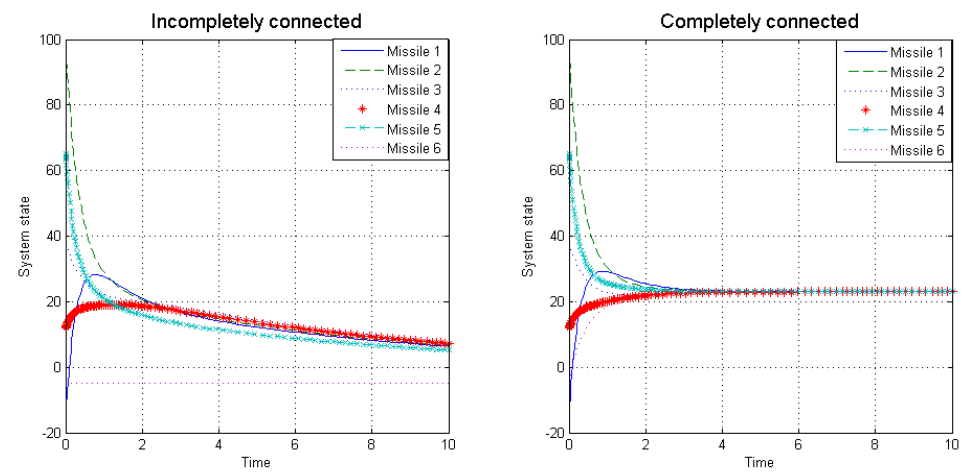

Fig.6 The variety of six missiles' states tend to be consistent

From the simulation results in Fig.6, it can be seen that the connectivity of the network will affect the state of entire missile formation. When attacked, the system of missile network has ability to achieve coherence in state; while not, the system converges to a stable state. In conclusion that the missile network system designed have good invulnerability.

Coope rative guidance simulation. Assumed that the target is stationary at $(0,0)$, the simulation step is $0.01 \mathrm{~s}$, the available load of the missile is $5 \mathrm{~g}, \mathrm{~g}=9.81 \mathrm{~m} / \mathrm{s}$, proportional coefficient is set as three, $\mathrm{K}=3$. The initial parameters of the 6 missile are shown in Table 1 .

Table 1 The initial parame ters of the 6 missiles

\begin{tabular}{cccc}
\hline Missile & Initial position $(\mathrm{m})$ & Flight velocity $(\mathrm{m} / \mathrm{s})$ & trajectory deflection angle $\left({ }^{\circ}\right)$ \\
\hline 1 & $(-6894.00,-5785.00)$ & 280.00 & 72.00 \\
2 & $(-3249.00,-8927.00)$ & 320.00 & 95.00 \\
3 & $(2329.00,-8693.00)$ & 260.00 & 110.00 \\
4 & $(3223.00,-7024.00)$ & 300.00 & 75.00 \\
5 & $(-3124.00,-7453.00)$ & 280.00 & 80.00 \\
6 & $(-5821.00,-5321.00)$ & 300.00 & 100.00 \\
\hline
\end{tabular}

Expected guidance time $T_{d}$ is set as $36 \mathrm{~s}$ in this simulation. The missile terminal guidance result is obtained by using the distributed cooperative strategy in two cases, which is not incompletely connected and completely connected in the missile network.
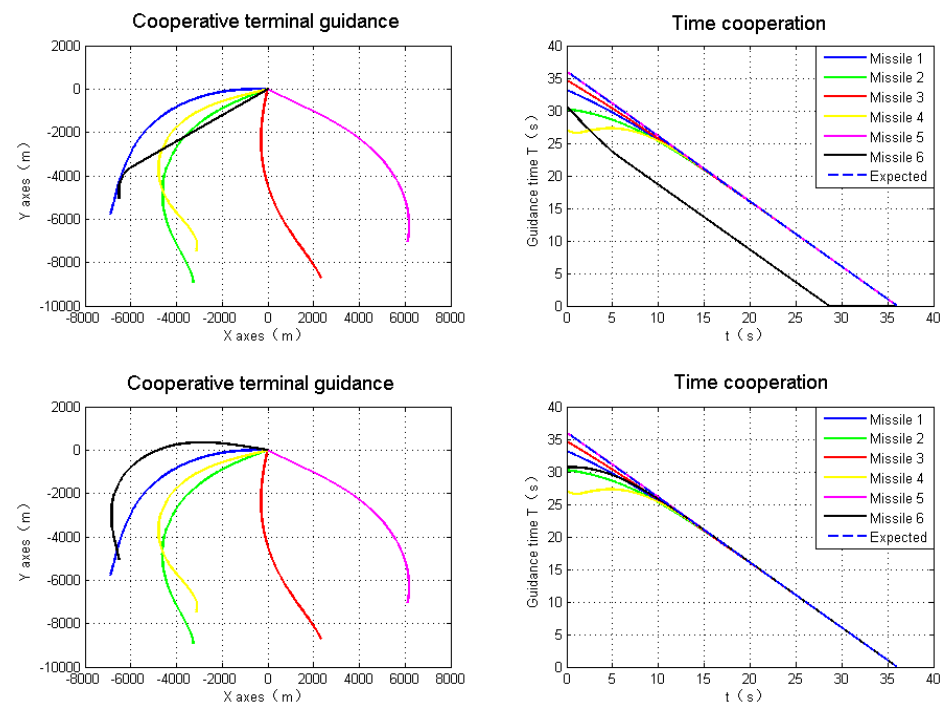

Fig.7 Distributed cooperative guidance result

From the distributed cooperative guidance result in Fig. 7, 6 missiles are capable of hitting the target with time not being cooperative when missile network being attacked, the $6^{\text {th }}$ missile hits the target prematurely at approximately $28^{\text {th }}$ second, while other missiles cost about 36.06 seconds. Nevertheless, all missiles have hit the target cooperatively when network in normal operation 
costing 36.06 seconds.

\section{Conclusion}

In this study, we add time correction on the basis of the pure proportional navigation law applied to solve the problem of multi-missile distributed cooperative guidance based on dynamic network. In order to demonstrate the success of the method, it was applied to two different situations that missile network completely connected or attacked .It is clearly seen from the results that missile network have good invulnerability and the missile network coordinate guidance control method is of effectiveness and feasibility.

\section{Acknowledge ments}

This work is financially supported by the National Natural Science Fund of China (61164015), Province Natural Science Fund of JiangXi (20151BAB207043) and Province Graduate Innovation Fund of JiangXi (YC2014-S393).

\section{References}

[1] Wei Ren, Yongcan Cao. Distributed Coordination of Multi-agent Networks[M].Springer London.2011

[2] Zhendong,Sun Jie Huang. A note on connectivity of multi-agent systems with proximity graphs and linear feedback protocol[J]. Automatica, 2009,45(8):1953-1956.

[3] Y.A. Zhang, D.H. Yu, Y.G Zhang. An impact-time-control guidance law for multi-missiles[J].Intelligent Computing and Intelligent Systems, 2009(2): 430-434.

[4] I.S. Jeon, J.I. Lee. Homing guidance law for cooperative attack of multiple missiles [J].Journal of Guidance, Control and Dynamics,2010,33(1):275-280.

[5] S.Y. Zhao, R. Zhou, Wei Chen,etc. Design of time-constrained guidance laws via virtual leader approach [J]. Chinese Journal of Aeronautics, 2010, 23(1):103-108.

[6] X.Sun, Y.Q. Xia. Optimal guidance law for cooperative attack of multiple missilesbased on optimal control theory [J]. International Journal of Control, 2012, 85(8): 1063-1070.

[7] Wang B., Tang H. W., Guo C. H., et al. Entropy Optimization of Scale-Free Networks' Robustness to Random Failures. Physica A, 2006, 363(2): 591 - 596.

[8] JEON I S, LEE J I, TAHK M J. Impact-Time-Control Guidance Law for Anti-Ship Missiles [J]. IEEE Transactions on Control Systems Technology, 2006, 14(2):260-26

[9] JUNG B,KIM Y.Guidance law for anti-ship missiles using impact time[C]. AIAA Guidance,Navigation, and Control Conference and Exhibit. USA: AIAA,2006:1-13

[10] Newnan M.E.J.,Strogatz S.H.and Watts D. J. Random Graphs with Arbitrary Degree Distributions and their Applications. Physical Review E. 2001, 64026118:1-17. 